\title{
Subnotificação de Doenças Transmitidas por Alimentos em Januária-MG
}

\author{
Underreporting of Foodborne Diseases in Januária-MG
}

\author{
Juciara Aparecida da Silva Oliveira ${ }^{\text {a }}$ Luiz Carlos Ferreira*a
}

anstituto Federal Norte de Minas Gerais. MG, Brasil.

*E-mail: luiz.ferreira@ifnmg.edu.br

\begin{abstract}
Resumo
As Doenças Transmitidas por Alimentos (DTA) são causadas pela ingestão de alimentos ou bebidas contaminados, podendo afetar a saúde do consumidor. Este trabalho objetivou analisar os casos de notificação de Doenças Transmitidas por Alimentos no município de Januária-MG. Foi utilizado como base de dados o Sistema de Informação de Agravos de Notificação (SINAN) do Ministério da Saúde, além de informações obtidas na Secretaria Municipal de Saúde do Município de Januária. Foram utilizadas séries históricas dos casos de DTA, no município de Januária. A análise descritiva da base de dados do SINAN foi referente ao período entre 2007 a 2017. Também foi aplicado um questionário aos profissionais de saúde e ao público atendido pelas Unidades Básicas de Saúde sobre questões relacionadas às doenças transmitidas por alimentos. Foi possível observar que muitos dos dados constantes nas fichas de notificação estavam repetidos de um ano para o outro, ficando evidente a precária qualidade dos dados disponíveis de domínio público. No município de Januária, é evidente a baixa qualidade dos dados sobre notificações de DTA, revelando um quadro elevado de subnotificações. Despreparo, descuido ou negligência no preenchimento e encaminhamento das fichas de notificações podem estar relacionados com o reduzido número de notificações de DTA no município.
\end{abstract}

Palavras-chave: DTA. Notificação. Informação.

\begin{abstract}
Foodborne Diseases (DTA) are caused by the ingestion of contaminated food or beverages, which can affect the consumer's health. This study aimed to analyze the cases of notification of Foodborne Diseases in the municipality of Januária-MG. The Ministry of Health's Notifiable Diseases Information System (SINAN) was used as a database, in addition to information obtained from the Municipal Health Secretariat of the Municipality of Januária. Historical series of DTA cases in the municipality of Januária were used. The descriptive analysis of the SINAN database was for the period from 2007 to 2017. A questionnaire was also applied to health professionals and the public served by basic health units on issues related to foodborne diseases. It was possible to observe that many of the data contained in the notification forms were repeated from one year to the next, making evident the precarious quality of the data available in the public domain. In the municipality of Januária, the low quality of data on DTA notifications is evident, revealing a high level of underreporting. Unpreparedness, carelessness or negligence in filling and forwarding notification forms may be related to the reduced number of DTA notifications in the municipality.
\end{abstract}

Keywords: DTA. Notification. Information.

\section{Introdução}

A incidência de doenças veiculadas por alimentos cresce a cada ano no Brasil, porém se estima que um número elevado de casos não é notificado (SOUZA et al., 2021). No Brasil, no período entre 2016 a 2019 foi registrado um total de 626 surtos por ano de doenças transmitidas por água e alimentos, que acometeram 37.247 pessoas, sendo registrados 38 óbitos (BRASIL, 2020).

Todo surto de DTA deve ser notificado às autoridades locais de saúde e investigado, imediatamente, sendo que a Unidade de Saúde notificadora deve utilizar a ficha de notificação/investigação do SINAN, sendo que a mesma vem agregada com as instruções para o correto preenchimento, encaminhando-a para ser processada, conforme o fluxo estabelecido pela Secretaria Municipal de Saúde (BRASIL,
2010).

Dimensionar o total de casos de doenças transmitidas por alimentos é difícil, principalmente, em função da dificuldade na obtenção de dados precisos e a subnotificação pelos serviços de vigilância epidemiológica. Entretanto, a divulgação dessas informações pode contribuir com ações preventivas e regulatórias, otimizando a aplicação de recursos públicos e racionalizando políticas de saúde (SOARES, 2019).

A falta de notificação, aspectos culturais e a vulnerabilidade da população podem ser fatores relevantes para a ocorrência de um número relativamente grande de casos de DTA. Sendo assim, este estudo teve como objetivo avaliar o grau de subnotificações de Doenças Transmitidas por Alimentos no município de Januária-MG. 


\section{Desenvolvimento}

\subsection{Metodologia}

O estudo foi descritivo, quantitativo e retrospectivo, sendo utilizados dados provenientes do Sistema de Informação de Agravos de Notificação (SINAN) do Ministério da Saúde e informações obtidas da Secretaria Municipal de Saúde do município de Januária. Em complementação aos dados de domínio público, foram aplicados questionários aos enfermeiros e usuários das Unidades Básicas de Saúde da cidade de Januária, visando coletar informações sobre as notificações de DTA. Os formulários não foram submetidos a um Comitê de Ética por se tratar de formulários eletrônicos anônimos Google Forms.

Foram utilizadas séries históricas, dos casos de DTA, no município de Januária. A análise descritiva da base de dados do SINAN foi referente ao período que compreende entre 2007 a 2017. Da análise dos dados coletados foi determinado o grau de notificação/subnotificação de DTA no município de Januária.

\subsection{Discussão}

O município de Januária-MG, com população estimada para 2020 de 67.852 pessoas (IBGE, 2021), possui 24 Unidades Básicas de Saúde. Uma Unidade de Saúde se caracteriza por realizar um conjunto de ações de saúde, em âmbito individual e coletivo, que abrange a promoção e a proteção da saúde, a prevenção de agravos, o diagnóstico, o tratamento, a reabilitação, a redução de danos e a manutenção da saúde com o objetivo de desenvolver uma atenção integral, que impacte a situação de saúde e autonomia das pessoas e nos determinantes e condicionantes de saúde das coletividades (BRASIL, 2011).

Toda Unidade Básica de Saúde do município de Januária deve preencher, semanalmente, a ficha de notificação de doenças e agravos e enviar para o departamento de epidemiologia/imunização, porém, de acordo com dados da Secretaria Municipal de Saúde, apenas 13 Unidades de Saúde entregaram as fichas de notificação no ano de 2016, não sendo registrado nenhum relato de caso de DTA ou mesmo de doenças diarreicas aguda. A diarreia juntamente com a dor de estomacal, vômito e febre são sintomas de DTA, sendo que muitos casos de diarreia são causados por água ou alimentos contaminados.

Foi possível observar que muitos dos dados, constantes nas fichas de notificação, estavam repetidos de um ano para o outro, ficando evidente a precária qualidade dos dados disponíveis de domínio público. Segundo a Política Nacional de Atenção Básica, são atribuições comuns a todos os profissionais, membros das equipes de saúde básica, realizar busca ativa e notificar doenças e agravos de notificação compulsória e de outros agravos e situações de importância local (BRASIL, 2011).

No ano de 2017, 19 Unidades de Saúde enviaram as fichas de notificação sem casos suspeitos de DTA, porém com 7 casos de doença diarreica aguda. Neste mesmo ano, foi verificada inconsistência nos dados que pode ser observada, na maioria das fichas de notificação, tornando confusa a interpretação dos registros apresentados, deixando evidente o descaso e a falta de atenção no registro dos dados fornecidos.

A falta de cuidado, no registro dos dados, é muito evidente quando são avaliados os dados referentes ao número de entrevistas realizadas, número de visitas realizadas, número de notificações e número de casos investigados. Em julho de 2017 foram realizadas um total de 85 entrevistas, com 7 casos suspeitos de doença diarreica aguda registrados, já em agosto de 2017, o número de entrevistas realizadas subiu para 632 , um aumento de mais de $700 \%$ em relação ao mês anterior, sendo que, mesmo com esse expressivo aumento no número de entrevistas realizadas e com 1.385 visitas realizadas, foram registrados, no mês de agosto de 2017, o mesmo número de 7 casos de doença diarreica aguda em relação ao mês anterior, sendo a mesma Unidade de Saúde responsável pelo registro.

De acordo com dados do SINAN, no município de JanuáriaMG, no período entre 2007 a 2017 foram notificados somente dois surtos de DTA (Quadro 1). Mesmo sendo apenas duas notificações de surto de DTA e não sendo possível identificar o agente etiológico, foram 29 pessoas doentes. Embora seja crescente o número de notificações, a subnotificação e falhas nas etapas de investigação comprometem a geração de informações, que orientem as pesquisas laboratoriais dos possíveis agentes envolvidos, o que tem contribuído com o elevado número de processos não conclusivos (DIAS et al., 2011).

Quadro 1 - Dados dos surtos de DTA notificados no município de Januária-MG no período de 2007-2017

\begin{tabular}{|c|c|c|}
\hline \multirow{2}{*}{ Dados do surto } & \multicolumn{2}{|c|}{ Ano } \\
\hline & 2008 & 2011 \\
\hline Mês & Abril & Agosto \\
\hline Agente etiológico do surto & Inconclusivo & Ignorado \\
\hline Alimento causador do surto & Inconclusivo & Ignorado \\
\hline Local de ocorrência do surto & Outras Instituições (alojamento, trabalho) & Outras \\
\hline $\mathrm{N}^{\mathrm{o}}$ total de casos expostos & 139 & 6 \\
\hline $\mathrm{N}^{\mathrm{o}}$ total de doentes & 23 & 6 \\
\hline $\mathrm{N}^{\mathrm{o}}$ de óbitos & 0 & 0 \\
\hline Critério de confirmação do surto & Inconclusivo & Laboratorial clínico \\
\hline
\end{tabular}


Nos dados obtidos dos questionários aplicados ao público atendido pelas Unidades Básicas de Saúde, da cidade de Januária, é possível observar que, do total de entrevistados, $75 \%$ afirmaram já terem se sentido mal ao ingerir algum tipo de alimento e mais de $80 \%$ tiveram diarreia que pode estar associada à ingestão de alimentos ou água contaminada. Apenas 17\% dos entrevistados declararam que procuraram a Unidade de Saúde e $83 \%$ ficaram em casa mesmo tendo sintomas de DTA. Portanto, é evidente que existe um índice elevado de subnotificação, quando comparados os dados oficiais com os resultados dos questionários aplicados ao público atendido pelas Unidades Básicas de Saúde. Mais de $60 \%$ dos entrevistados confirmaram não terem feito nenhum exame complementar.

Diante dos resultados dos questionários, é possível constatar que as pessoas conhecem a importância das DTA, porém não sabem as consequências que essas podem ocasionar. A deficiência de informações, a resistência em busca atendimento adequada e a falta de interesse podem promover graves consequências para o município, uma vez que as DTA podem causar danos à economia em função do aumento dos gastos com internações que poderiam ser evitadas, no caso de diagnósticos corretos, com consequente tratamento adequado.

Das respostas aos questionários aplicados aos enfermeiros das Unidades Básicas de Saúde, da cidade de Januária, foi possível verificar que os profissionais de enfermagem, apesar de considerarem de grande importância as DTA, afirmaram que são insuficientes as informações prestadas pelo município sobre as DTA.

\section{Conclusão}

É elevado o índice de subnotificação de DTA no município de Januária, o que pode estar relacionado à desinformação da população quanto aos riscos representados por esse tipo de doença. É evidente a baixa qualidade dos dados sobre notificações de DTA, revelando um quadro elevado de subnotificações. Despreparo, descuido ou negligência no preenchimento e encaminhamento das fichas de notificações podem estar relacionadas com o reduzido número de notificações de DTA no município.

A falta de informação da população sobre as consequências resultantes de casos de DTA reduz, significativamente, a procura por Unidades Básicas de Saúde para o diagnóstico e tratamento adequado. Entretanto, a falta de notificação dos casos que chegam ao sistema de saúde reforça o panorama de desinteresse e subestimação dos riscos representados pelas DTA para saúde da população.

Faz-se necessário a conscientização da população e, principalmente, dos profissionais da área da saúde, no intuito de incentivar a busca por diagnóstico e tratamento, assim como do aprimoramento dos procedimentos de notificação de DTA, especialmente, no que se refere ao correto preenchimento das fichas de notificação.

\section{Referências}

BRASIL. Ministério da Saúde. Secretaria de Vigilância em Saúde. Portaria $n^{\circ}$ 2.472, 31 de agosto de 2010. Brasília: MS, 2010.

BRASIL. Ministério da Saúde. Política nacional de atenção básica. Brasília: MS, 2011.

BRASIL. Ministério da Saúde. Secretaria de Vigilância em Saúde. Bol. Epidemiol., v.51, n,32, 2020.

BRASIL. Ministério da Saúde. SINAN (Sistema de Informação de Agravos de Notificação). Disponível em: http://portalsinan. saude.gov.br/sinan-net. Acesso em: 26 maio 2021.

DIAS, R.S.; LEAL BERNARDES, A.F.; ZUCCOLI, P.C. A importância do processo de investigação na elucidação de surtos de Doenças Transmitidas por Alimentos (DTA). NBC, v.1, n.2, p.17-23, 2011.

IBGE. Instituto Brasileiro de Geografia e Estatística. Cidades e estados. Disponível em: https://ibge.gov.br/cidades-e-estados/ $\mathrm{mg} /$ januaria.html. Acesso em: 24 maio 2021.

SOARES, M.V. Intoxicações por Alimentos no Brasil Registradas pelo SINITOX Entre 1999 e 2017. Uniciências, v. 23, n. 1, p. 5256, 2019. doi:10.17921/1415-5141.2019v23n1p52-56.

SOUZA, J.F.; SOUZA, A.C.F.; COSTA, F.N. O. Estudo retrospectivo de surtos de doenças veiculadas por alimentos, na região nordeste e Estado do Maranhão, no período de 2007 a 2019. Res. Soc. Dev., v.10, n.1, p.1-8, 2021. doi: 10.33448/rsdv10i1.11728. 